\title{
Hypoxia and perfusion in breast cancer: simultaneous assessment using PET/MR imaging
}

\author{
Julia C. Carmona-Bozo ${ }^{1} \cdot$ Roido Manavaki $^{1}$ - Ramona Woitek ${ }^{1,2}$ - Turid Torheim ${ }^{3}$ - Gabrielle C. Baxter ${ }^{1}$. \\ Corradina Caracò $^{1}$ • Elena Provenzano ${ }^{3,4}$ • Martin J. Graves ${ }^{1,5} \cdot$ Tim D. Fryer $^{6}$ • Andrew J. Patterson ${ }^{1,5}$. \\ Fiona J. Gilbert ${ }^{1}$ (10)
}

Received: 26 February 2020 / Revised: 12 May 2020 / Accepted: 3 July 2020 / Published online: 28 July 2020

(C) The Author(s) 2020

\begin{abstract}
Objectives Hypoxia is associated with poor prognosis and treatment resistance in breast cancer. However, the temporally variant nature of hypoxia can complicate interpretation of imaging findings. We explored the relationship between hypoxia and vascular function in breast tumours through combined ${ }^{18} \mathrm{~F}$-fluoromisonidazole $\left({ }^{18} \mathrm{~F}\right.$-FMISO) PET/MRI, with simultaneous assessment circumventing the effect of temporal variation in hypoxia and perfusion.

Methods Women with histologically confirmed, primary breast cancer underwent a simultaneous ${ }^{18} \mathrm{~F}$-FMISO-PET/MR examination. Tumour hypoxia was assessed using influx rate constant $K_{\mathrm{i}}$ and hypoxic fractions (\%HF), while parameters of vascular function $\left(K^{\text {trans }}, k_{\mathrm{ep}}, v_{\mathrm{e}}, v_{\mathrm{p}}\right)$ and cellularity (ADC) were derived from dynamic contrast-enhanced (DCE) and diffusion-weighted (DW)-MRI, respectively. Additional correlates included histological subtype, grade and size. Relationships between imaging variables were assessed using Pearson correlation $(r)$.

Results Twenty-nine women with 32 lesions were assessed. Hypoxic fractions $>1 \%$ were observed in $6 / 32(19 \%)$ cancers, while 18/32 (56\%) tumours showed a \%HF of zero. The presence of hypoxia in lesions was independent of histological subtype or grade. Mean tumour $K^{\text {trans }}$ correlated negatively with $K_{\mathrm{i}}(r=-0.38, p=0.04)$ and \%HF $(r=-0.33, p=0.04)$, though parametric maps exhibited intratumoural heterogeneity with hypoxic regions colocalising with both hypo- and hyperperfused areas. No correlation was observed between ADC and DCE-MRI or PET parameters. \%HF correlated positively with lesion size $(r=0.63, p=0.001)$.

Conclusion Hypoxia measured by ${ }^{18} \mathrm{~F}$-FMISO-PET correlated negatively with $K^{\text {trans }}$ from DCE-MRI, supporting the hypothesis of perfusion-driven hypoxia in breast cancer. Intratumoural hypoxia-perfusion relationships were heterogeneous, suggesting that combined assessment may be needed for disease characterisation, which could be achieved using simultaneous multimodality imaging.
\end{abstract}

Electronic supplementary material The online version of this article (https://doi.org/10.1007/s00330-020-07067-2) contains supplementary material, which is available to authorized users.

Fiona J. Gilbert

fjg28@medschl.cam.ac.uk

1 Department of Radiology, School of Clinical Medicine, University of Cambridge, Box 218, Cambridge Biomedical Campus,

Cambridge CB2 0QQ, UK

2 Department of Biomedical Imaging and Image-Guided Therapy, Medical University of Vienna, Währinger Gürtel 18-20, 1090 Vienna, Austria

3 Cancer Research UK - Cambridge Institute, University of Cambridge, Li Ka Shing Centre, Robinson Way, Cambridge CB2 ORE, UK
4 Cambridge Breast Unit, Cambridge University Hospitals NHS Foundation Trust, Box 97, Cambridge Biomedical Campus, Cambridge CB2 0QQ, UK

5 MRIS Unit, Cambridge University Hospitals NHS Foundation Trust, Box 162, Cambridge Biomedical Campus, Cambridge CB2 0QQ, UK

6 Wolfson Brain Imaging Centre, Department of Clinical Neurosciences, School of Clinical Medicine, University of Cambridge, Box 65, Cambridge Biomedical Campus, Cambridge CB2 0QQ, UK 


\section{Key Points}

- At the tumour level, hypoxia measured by ${ }^{18}$ F-FMISO-PET was negatively correlated with perfusion measured by DCE-MRI, which supports the hypothesis of perfusion-driven hypoxia in breast cancer.

- No associations were observed between 18F-FMISO-PET parameters and tumour histology or grade, but tumour hypoxic fractions increased with lesion size.

- Intratumoural hypoxia-perfusion relationships were heterogeneous, suggesting that the combined hypoxia-perfusion status of tumours may need to be considered for disease characterisation, which can be achieved via simultaneous multimodality imaging as reported here.

Keywords PET/MRI $\cdot$ Hypoxia $\cdot$ Perfusion $\cdot$ Breast cancer

$\begin{array}{ll}\text { Abbreviations } \\ \text { \% HF } & \text { Percentage hypoxic fraction } \\ { }^{18} \mathrm{~F}-\mathrm{FMISO} & { }^{18} \mathrm{~F} \text {-fluoromisonidazole } \\ \text { ADC } & \text { Apparent diffusion coefficient }\left(\mathrm{mm}^{2} / \mathrm{s}\right) \\ k_{\mathrm{ep}} & \text { Contrast efflux rate constant }\left(\mathrm{min}^{-1}\right) \\ K_{\mathrm{i}} & \text { Tracer influx rate constant }\left(\mathrm{mL} / \mathrm{cm}^{3} / \mathrm{min}\right) \\ K^{\text {trans }} & \text { Contrast influx transfer rate } \\ & \text { constant }(\mathrm{mL} / \mathrm{g} / \mathrm{min}) \\ \mathrm{SUV} & \text { Standardised uptake value }(\mathrm{g} / \mathrm{mL}) \\ T_{\max } / M & \text { Maximum tumour-to-muscle ratio } \\ T_{\max } / P & \text { Maximum tumour-to-plasma ratio } \\ v_{\mathrm{e}} & \text { Extravascular-extracellular volume fraction } \\ v_{\mathrm{p}} & \text { Plasma volume fraction }\end{array}$

\section{Introduction}

Hypoxia is a common characteristic of the tumour microenvironment and arises due to avid metabolism and poor perfusion as a result of the structurally and functionally aberrant microcirculation found in tumours [1]. In breast cancer, the presence of hypoxia has been confirmed with $\mathrm{pO}_{2}$ histography and occurs irrespective of histological type, molecular subtype, grade or patient characteristics $[2,3]$. In vitro studies have shown that hypoxia promotes a dedifferentiated phenotype in ductal carcinoma in situ [4] and downregulates the expression and function of oestrogen receptor- $\alpha(E R \alpha)$ [5]. Several clinical and preclinical studies in breast cancer have demonstrated that overexpression of hypoxia-related proteins is associated with an aggressive phenotype, poor prognosis and resistance to treatment [6-8].

Although tumour hypoxia can be broadly categorised as diffusion or perfusion limited, it is generally accepted that the tumour microenvironment is a highly dynamic entity, exhibiting temporally varying perfusion patterns and heterogeneous oxygen-tension gradients [9]. Experimental evidence suggests that oxygen levels continually fluctuate owing to transient changes in perfusion [10]. These changing perfusion and oxygenation levels induce a variety of gene expression profiles resulting in a unique micromilieu that is pivotal for tumour growth and metastatic dissemination [11]. Given the temporal variation in oxygenation and perfusion within tumours, sequential multimodal imaging investigations may not always be effective in assessing the association between these parameters, as similar tumour status cannot be guaranteed between imaging sessions. Simultaneous assessment of the hypoxia and perfusion in tumours can mitigate confounders associated with the dynamic character of these processes, and thus allow additional pathophysiological characterisation of breast cancer.

Imaging methods, including positron emission tomography (PET) and magnetic resonance imaging (MRI), have been used for the non-invasive assessment of the tumour microenvironment. Dynamic contrast-enhanced (DCE) MRI has shown utility in characterising tumour perfusion and vascular permeability in clinical studies [12], while diffusion-weighted imaging (DWI) can provide surrogate measures of tumour cellular density [13]. PET with ${ }^{18} \mathrm{~F}$-labelled nitroimidazoles can provide specific measures of intracellular hypoxia [14]. In breast cancer, ${ }^{18} \mathrm{~F}$ fluoromisonidazole ( ${ }^{18} \mathrm{~F}$-FMISO) has been used for the evaluation of response to anti-angiogenic and HER2-targetted treatment $[15,16]$ and shown potential utility as a predictor of response to primary endocrine therapy $[17,18]$. Additionally, high ${ }^{18}$ F-FMISO uptake at baseline has been associated with shorter disease-free survival [18] and disease-specific death [19].

Despite the intrinsic link between tumour hypoxia and perfusion, multimodal imaging approaches to characterise this aspect of cancer pathophysiology have been limited in the clinical setting [16, 19-24]. To effectively assess relationships between temporally varying microenvironment parameters, combined PET/MR imaging presents an attractive option as it permits examination of the tumour under the same physiologic conditions, while also conferring methodological advantages in the spatial registration of data from the two modalities.

The primary objective of this study was to examine the association between hypoxia and vascular function in patients with treatment-naïve breast cancer using simultaneous ${ }^{18} \mathrm{~F}$ FMISO-PET/MRI. To our knowledge, this is the first such study in breast cancer. 


\section{Materials and methods}

\section{Study participants}

Women aged $>18$ years with histologically confirmed primary breast cancer and a tumour diameter $>10 \mathrm{~mm}$ on mammography and/or ultrasound were eligible for the study (February 2017 to November 2018). Pregnancy, lactation, previous surgery or radiotherapy for cancer or benign breast disease, inadequate renal function and contraindications to MRI were exclusion criteria for the study. The research was approved by a National Research Ethics Committee (14/EE/0145). All study participants provided written informed consent before PET/ MRI examination.

\section{PET/MRI acquisition}

Participants underwent a 60-min simultaneous PET/MR scan of the breasts in the prone position on a SIGNA PET/MR scanner (GE Healthcare), using a 16-channel bilateral breast array (RAPID Biomedical) $120 \mathrm{~min}$ (median [range], 120.2 [119.8-127.5] min) after injection of $306 \pm 14 \mathrm{MBq}^{18} \mathrm{~F}$-FMISO. The uptake period post injection (p.i.) was used to enhance hypoxic-to-normoxic tissue contrast and allow the free ${ }^{18} \mathrm{~F}$-FMISO concentrations in tissue and blood to reach equilibrium [25, 26], a requirement for influx rate constant $\left(K_{\mathrm{i}}\right)$ determination by Patlak analysis [27].

PET: Emission data from 120 to $180 \mathrm{~min}$ p.i. $(12 \times 5-\mathrm{min}$ frames) were reconstructed using time-of-flight orderedsubsets expectation-maximisation (TOF-OSEM) with 4 iterations and 28 subsets (Supplemental Methods I). Plasma radioactivity concentration from two venous blood samples, acquired immediately before and after PET/MR acquisition, was used to scale a ${ }^{18} \mathrm{~F}$-FMISO population-based arterial input function (AIF) derived from existing data, permitting calculation of $K_{\mathrm{i}}$ [28] (Supplemental Methods II; Supplemental Fig. 1; [29-31]).

MRI: The MRI protocol involved a 2-point Dixon sequence for PET attenuation correction, $\mathrm{T}_{1}$ - and $\mathrm{T}_{2}$-weighted images, DWI, and a DCE series. Sequences were also acquired to measure $\mathrm{B}_{1}{ }^{+}$transmission-field non-uniformity, using a Bloch-Siegert method, and baseline $T_{1}\left(T_{10}\right)$ as required for the pharmacokinetic analysis of DCE-MRI data [32]. DCEMRI acquisition involved five pre-contrast images, followed by 43 phases after intravenous bolus injection of $0.1 \mathrm{mmol} / \mathrm{kg}$ of Gadovist (Bayer Healthcare). MRI sequence details are given in Supplemental Table 1.

\section{Image analysis}

Tumour regions were manually delineated in OsiriX, version 8.0.2 (Pixmeo SARL), by three radiologists in
Table 1 Clinical characteristics for the patient population $(n=29)$

\begin{tabular}{|c|c|}
\hline Characteristic & $n(\%)$ \\
\hline Age at diagnosis (years) ${ }^{\mathrm{a}}$ & $57[37-78]$ \\
\hline Lesions & 32 \\
\hline Pathological size $(\mathrm{mm})^{\mathrm{a}, \mathrm{b}}$ & 26 [10-142] \\
\hline \multicolumn{2}{|c|}{ Lesion longest diameter on MRI } \\
\hline$\leq 20 \mathrm{~mm}$ & $10(31)$ \\
\hline$>20 \mathrm{~mm}$ & $22(69)$ \\
\hline \multicolumn{2}{|l|}{ Histopathological subtype } \\
\hline Ductal (IDC) & $21(66)$ \\
\hline Lobular (ILC) & $6(19)$ \\
\hline Mucinous (IMC) & $2(6)$ \\
\hline Mixed $^{\mathrm{c}}$ & $3(9)$ \\
\hline \multicolumn{2}{|l|}{ Histological grade ${ }^{\mathrm{d}}$} \\
\hline 1 & $3(9)$ \\
\hline 2 & $16(50)$ \\
\hline 3 & $13(41)$ \\
\hline \multicolumn{2}{|l|}{ Hormone-receptor status ${ }^{\mathrm{e}}$} \\
\hline Positive (ER or PR) & $31(97)$ \\
\hline Negative & $1(3)$ \\
\hline \multicolumn{2}{|l|}{ HER2 status ${ }^{\mathrm{f}}$} \\
\hline Positive & $7(22)$ \\
\hline Negative & $25(78)$ \\
\hline
\end{tabular}

$E R$ oestrogen receptor, $P R$ progesterone receptor, HER2 human epidermal growth factor receptor 2

${ }^{\mathrm{a}}$ Data presented as median [range]

${ }^{\mathrm{b}}$ Pathological size measured on tumour specimens from patients undergoing primary surgery $(n=21)$

${ }^{\mathrm{c}}$ Invasive carcinomas with presence of both lobular and ductal components on histology

${ }^{\mathrm{d}}$ Nottingham combined histologic grade

${ }^{\mathrm{e}}$ Tumours classified as ER or PR-positive, if $>10 \%$ of the cells demonstrated nuclear staining by immunohistochemistry

${ }^{\mathrm{f}}$ Tumours classified as HER2-positive, if they scored 3+ on immunohistochemistry or if they carried gene amplification as detected by fluorescence in situ hybridisation (FISH)

consensus (1, 3 and $>20$ years of experience in breast MRI). Regions were drawn on the peak-enhancing volumes of the DCE-MRI series on all contiguous axial sections encompassing the invasive part of the tumour and including multifocal/multicentric disease (Supplemental Methods III). Synchronous bilateral cancers were regarded as independent lesions [33].

DCE-MRI: Pharmacokinetic analysis of the DCE-MRI series was performed in MIStar, version 3.2.63 (Apollo Medical Imaging), using the extended Tofts' model [34] to calculate contrast influx rate constant, $K^{\text {trans }}$; efflux-rate constant, $k_{\mathrm{ep}}$; extravascular-extracellular volume fraction, $v_{\mathrm{e}}$; and plasma volume fraction, $v_{\mathrm{p}}$ (Supplemental Methods III). 

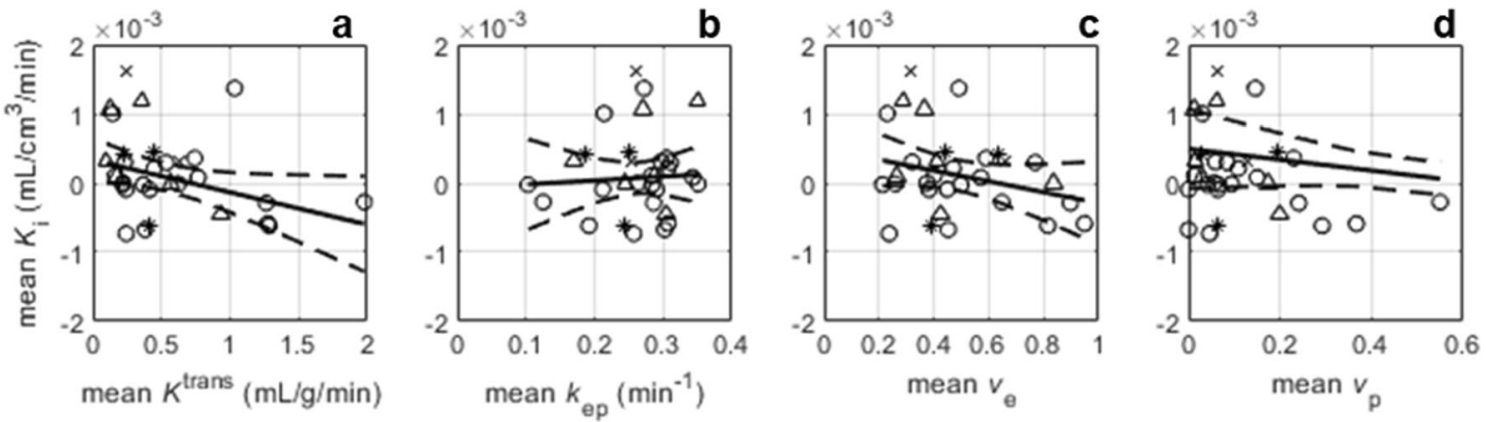

$\circ$ IDC $\quad \triangle \quad$ ILC $\quad \times \quad$ IMC $\quad * \quad$ Mixed
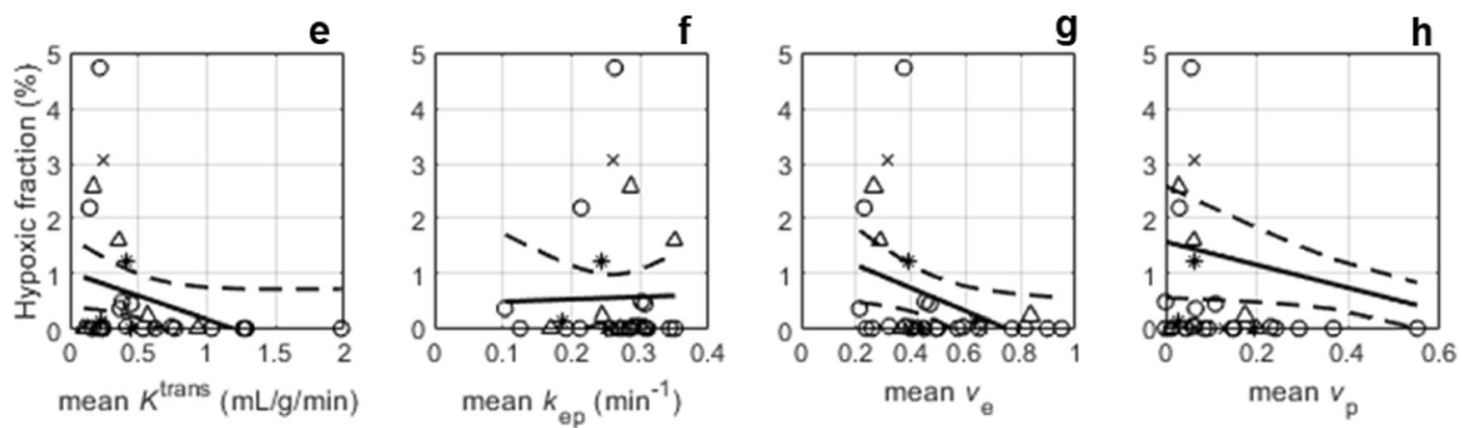

Fig. $1{ }^{18}$ F-FMISO-PET $K_{\mathrm{i}}$ and hypoxic fraction (\%) vs. the following DCE-MRI parameters: (a, e) contrast influx rate, $K^{\text {trans }}(\mathrm{mL} / \mathrm{g} / \mathrm{min}) ;(\mathbf{b}, \mathbf{f})$ contrast efflux rate, $k_{\mathrm{ep}}\left(\mathrm{min}^{-1}\right) ;(\mathbf{c}, \mathbf{g})$ fractional volume of extravascular-

DWI: Calculation of apparent diffusion coefficient (ADC) maps was performed in OsiriX, using $b$ values of 0 and $900 \mathrm{~s} /$ $\mathrm{mm}^{2}$. Mean lesion ADC was calculated by manually outlining whole tumour regions on the $b=900 \mathrm{~s} / \mathrm{mm}^{2}$ image (Supplemental Methods III; [35] ).

PET: Image frames from 150 to $180 \mathrm{~min}$ p.i. were averaged, rigidly registered to the peak-enhancing phase of the DCE-MRI series and subsequently employed for the determination of ${ }^{18} \mathrm{~F}$-FMISO uptake as mean and maximum standardised uptake values normalised by body weight $\left(\mathrm{SUV}_{\text {mean }}, \mathrm{SUV}_{\max }\right)$ and maximum tumour-to-plasma $\left(T_{\max } /\right.$ $P)$ and tumour-to-muscle $\left(T_{\max } / M\right)$ ratios within the regions defined on the DCE-MRI. The influx rate of ${ }^{18} \mathrm{~F}$-FMISO into the trapped (hypoxic) tissue compartment $\left(K_{\mathrm{i}}\right)$ was determined by Patlak-plot analysis, utilising all frames in the registered ${ }^{18} \mathrm{~F}$-FMISO series and the scaled population-based AIF. Hypoxic fractions $(\% \mathrm{HF})$ in tumour regions were calculated as the percentage of voxels with $K_{\mathrm{i}}$ values $>2 \times$ standard deviations (SD) of the mean $K_{\mathrm{i}}$ of normoxic muscle (Supplemental Methods III).

\section{Histology}

Histopathological information including tumour histological subtype, grade, oestrogen receptor (ER), progesterone receptor (PR) and human epidermal growth factor receptor-2 (HER2) status was obtained from core biopsies or surgical extracellular space, $v_{\mathrm{e}} ;(\mathbf{d}, \mathbf{h})$ plasma fractional volume, $v_{\mathrm{p}}$. IDC, invasive ductal carcinoma; ILC, invasive lobular carcinoma; IMC, invasive mucinous carcinoma; Mixed, carcinoma of mixed ductal and lobular type

tumour specimens. Cancers with positive ER or PR expression were classified as hormone-receptor (HR) positive.

\section{Statistics}

Statistical analysis was performed in IBM SPSS Statistics for MacOS, v25.0 (IBM Corp.) or Matlab 2016b. Continuous data were assessed for normality using the AndersonDarling test. Correlations between continuous variables were assessed using the Pearson correlation coefficient $(r) . t$ tests were used for comparison between means of two groups, and ANOVA when more than two groups were compared. Where data were not normally distributed, or normality could not be assessed, Mann-Whitney $U$ and Mood's median or KruskalWallis $H$ tests were employed for comparisons between two or more groups, respectively. $p$ values $<0.05$ were considered statistically significant.

\section{Results}

A total of 32 women were enrolled into the study. Two participants withdrew before the PET/MR examination. PET/MRI data and DCE-MRI data from two participants were excluded owing to inadequate acquisition of DCE-MRI and poor pharmacokinetic-model fitting respectively. In total, data from 29 participants with 32 biopsy-confirmed primary breast cancers 
were analysed. ADC calculations included data from 18 patients (19 lesions), who successfully completed the DWI examination.

Two thirds of the lesions $(21 / 32 ; 66 \%)$ were invasive ductal cancers (IDC). The majority of cancers $(29 / 32 ; 91 \%)$ were either grade 2 or 3. HR-positive expression was noted for $31 / 32(97 \%)$ lesions, with $24 / 32(77 \%)$ cancers being HER2-negative. Tumour characteristics are summarised in Table 1. Additional clinical information is provided in Supplemental Table 2.

\section{Relationship between ${ }^{18}$ F-FMISO-PET and DCE-MRI parameters}

Scatter plots indicating the relationships between DCE-MRI parameters and $K_{\mathrm{i}}$ or $\% \mathrm{HF}$ are illustrated in Fig. 1. An inverse relationship was observed between mean lesion $K_{\mathrm{i}}$ and $K^{\text {trans }}$, $v_{\mathrm{e}}$ and $v_{\mathrm{p}}$ (Fig. 1(a-d); Supplemental Fig. 2 [36]), which was statistically significant for $K_{\mathrm{i}}$ vs. $K^{\text {trans }}(r=-0.38, p=0.04)$, but not for $K_{\mathrm{i}}$ vs. $v_{\mathrm{e}}(r=-0.30, p=0.10)$ or $v_{\mathrm{p}}(r=-0.28, p=$ $0.12)$. Associations between $\% \mathrm{HF}$ and DCE-MRI parameters followed similar trends, also indicating a decrease in hypoxia with increasing $K^{\text {trans }}, v_{\mathrm{e}}$ and $v_{\mathrm{p}}$ (Fig. 1(e-h)). Statistically significant correlations were observed between $\% \mathrm{HF}$ and both $K^{\text {trans }}(r=-0.33, p=0.04)$ and $v_{\mathrm{e}}(r=-0.38, p=0.03)$. No correlation was observed between $k_{\mathrm{ep}}$ and either $K_{\mathrm{i}}(r=0.08$, $p=0.65)$ or $\% \mathrm{HF}(r=0.02, p=0.90)$.

Figure 2 presents axial slices through $K_{\mathrm{i}}$ and $K^{\text {trans }}$ parametric maps of four tumours of different histological subtypes, indicating heterogeneous spatial relationships between hypoxia and perfusion; other DCE-MRI parametric images are given in Supplemental Fig. 3.

\section{${ }^{18} \mathrm{~F}$-FMISO-PET and DCE-MRI parameters vs. tumour histology and grade}

Hypoxic fractions $>1 \%$ were observed in 6/32 (19\%) cancers with an additional 8/32 (25\%) lesions displaying hypoxic fractions greater than zero but less than $1 \%$; the remaining $18 / 32$ $(56 \%)$ tumours had a $\% \mathrm{HF}$ of zero. Dot plots of $\% \mathrm{HF}$ vs. tumour histological subtype and grade are presented in Fig. 3. $K_{\text {i }}$, $\% \mathrm{HF}$ and ${ }^{18}$ F-FMISO uptake parameters showed no significant difference between different histological subtypes or grades (Tables 2 and 3). Similarly, no significant differences were observed between histological groups or grades for the DCE-
Fig. 2 Axial images of four representative patients with: a invasive ductal carcinoma (IDC); b invasive lobular carcinoma (ILC); $\mathbf{c}$ invasive mucinous carcinoma (IMC); and d carcinoma of mixed ductal and lobular type (Mixed). (Left-toright) DCE-MRI image at peak enhancement; $K^{\text {trans }}$ map representing tumour perfusion for the lesion ROI overlaid on the peak-enhancing DCE-MRI image; $K_{\mathrm{i}}$ map representing tumour hypoxia for the lesion ROI overlaid on the peakenhancing DCE-MRI image; scatter plot and regression line of $K_{\mathrm{i}}$ vs. $K^{\text {trans }}$ voxel values within the tumour. $K^{\text {trans }}$, contrast influx rate $(\mathrm{mL} / \mathrm{g} / \mathrm{min}) ; K_{\mathrm{i}},{ }^{18} \mathrm{~F}$-FMISO influx rate $\left(\mathrm{mL} / \mathrm{cm}^{3} / \mathrm{min}\right)$

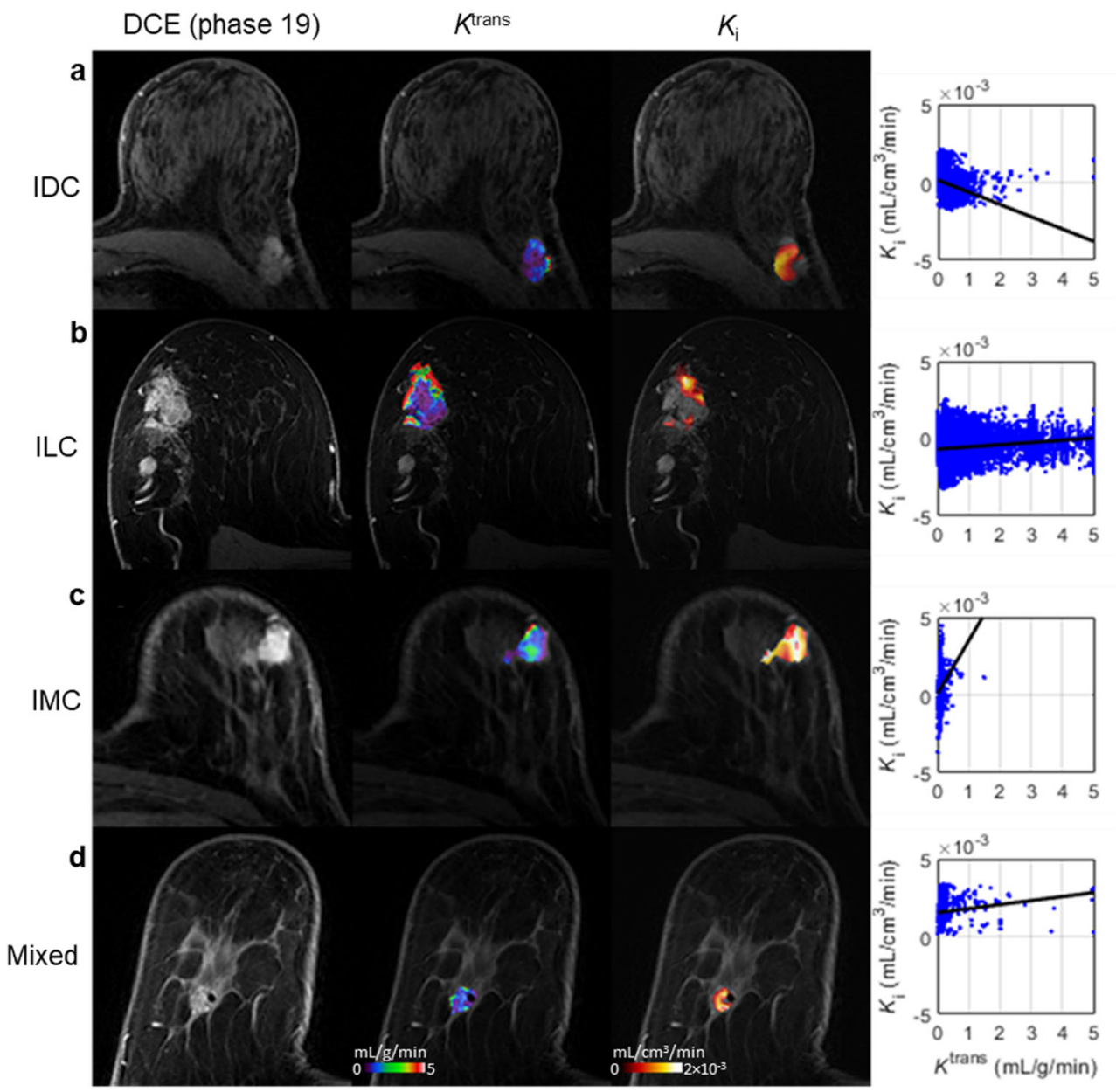


Fig. 3 Dot plots of hypoxic fraction (\%) by (a) histological type and (b) nuclear grade
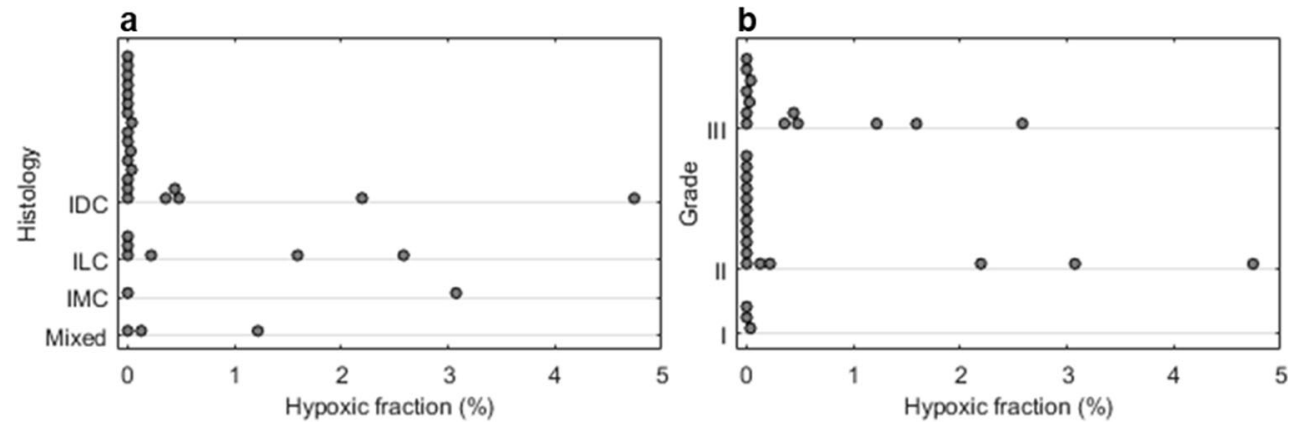

derived parameters (Tables 2 and 3), except for the efflux rate constant $k_{\text {ep }}$, which displayed a statistically significant difference among grade 2 and 3 cancers (median [range], 0.25 [0.13-0.34] vs. $\left.0.30[0.10-0.35] \mathrm{min}^{-1} ; p=0.01\right)$. Furthermore, analysis of hypoxia and $K^{\text {trans }}$ values in the most vascularised area of the tumour (hotspot on DCE-MRI) yielded no significant differences among different subtypes or grades (Supplemental Tables 3 and 4).

\section{Effect of tumour size on ${ }^{18}$ F-FMISO-PET and DCE-MRI parameters}

Table 4 presents correlations between imaging indices and tumour size as measured by longest diameter on MRI or pathological size. No or weak negative correlations were observed between tumour size and DCE-MRI parameters. Conversely, ${ }^{18}$ F-FMISO-PET parameters correlated positively with size;

Table 2 MRI and ${ }^{18}$ F-FMISO-PET parameters with respect to tumour histology. Data are presented as median [range] or mean \pm standard deviation (SD) as appropriate

\begin{tabular}{|c|c|c|c|c|c|}
\hline \multirow[t]{2}{*}{ Parameter } & \multicolumn{4}{|l|}{ Histology } & \multirow[t]{2}{*}{$p$ value } \\
\hline & IDC & ILC & Mixed & IMC & \\
\hline Lesions $(n=31)$ & 20 & 6 & 3 & 2 & \\
\hline$K^{\text {trans }}$ & $\begin{array}{l}0.43 \\
{[0.14-1.97]}\end{array}$ & $\begin{array}{l}0.26 \\
{[0.10-0.94]}\end{array}$ & $\begin{array}{l}0.41 \\
{[0.23-0.45]}\end{array}$ & $\begin{array}{l}0.44 \\
{[0.25-0.64]}\end{array}$ & $0.77^{\mathrm{a}}$ \\
\hline$k_{\mathrm{ep}}$ & $\begin{array}{l}0.26 \\
{[0.10-0.35]}\end{array}$ & $\begin{array}{l}0.28 \\
{[0.17-0.35]}\end{array}$ & $\begin{array}{l}0.25 \\
{[0.19-0.25]}\end{array}$ & $\begin{array}{l}0.26 \\
{[0.25-0.26]}\end{array}$ & $0.14^{\mathrm{a}}$ \\
\hline$v_{\mathrm{e}}$ & $\begin{array}{l}0.46 \\
{[0.21-0.95]}\end{array}$ & $\begin{array}{l}0.39 \\
{[0.26-0.84]}\end{array}$ & $\begin{array}{l}0.44 \\
{[0.39-0.64]}\end{array}$ & $\begin{array}{l}0.49 \\
{[0.31-0.66]}\end{array}$ & $0.30^{\mathrm{a}}$ \\
\hline$v_{\mathrm{p}}$ & $\begin{array}{l}0.08 \\
{[0-0.55]}\end{array}$ & $\begin{array}{l}0.05 \\
{[0.01-0.2]}\end{array}$ & $\begin{array}{l}0.06 \\
{[0.03-0.19]}\end{array}$ & $\begin{array}{l}0.09 \\
{[0.06-0.13]}\end{array}$ & $0.77^{\mathrm{a}}$ \\
\hline Lesions $(n=19)$ & 14 & 3 & 1 & 1 & \\
\hline $\operatorname{ADC}\left(\times 10^{-3}\right)$ & $\begin{array}{l}0.90 \\
{[0.42-1.55]}\end{array}$ & $\begin{array}{l}1.05 \\
{[0.84-1.28]}\end{array}$ & $\begin{array}{l}1.02 \\
{[-]}\end{array}$ & $\begin{array}{l}2.46 \\
{[-]}\end{array}$ & $0.51^{\mathrm{b}}$ \\
\hline Lesions $(n=32)$ & 21 & 6 & 3 & 2 & \\
\hline$K_{\mathrm{i}}\left(\times 10^{-3}\right)$ & $0.00 \pm 0.52$ & $0.37 \pm 0.65$ & $0.08 \pm 0.61$ & $0.97 \pm 0.91$ & $0.26^{\mathrm{c}}$ \\
\hline$\% \mathrm{HF}$ & $\begin{array}{l}0 \\
{[0-4.74]}\end{array}$ & $\begin{array}{l}0.10 \\
{[0-2.58]}\end{array}$ & $\begin{array}{l}0.13 \\
{[0-1.22]}\end{array}$ & $\begin{array}{l}1.54 \\
{[0-3.07]}\end{array}$ & $0.63^{\mathrm{a}}$ \\
\hline $\mathrm{SUV}_{\max }$ & $1.53 \pm 0.41$ & $1.77 \pm 0.16$ & $1.60 \pm 0.21$ & $1.25 \pm 0.12$ & $0.31^{\mathrm{c}}$ \\
\hline $\mathrm{SUV}_{\text {mean }}$ & $1.14 \pm 0.26$ & $1.27 \pm 0.18$ & $1.17 \pm 0.16$ & $1.07 \pm 0.15$ & $0.65^{\mathrm{c}}$ \\
\hline$T_{\max } / M$ & $1.02 \pm 0.24$ & $1.30 \pm 0.29$ & $1.09 \pm 0.22$ & $0.95 \pm 0.02$ & $0.12^{\mathrm{c}}$ \\
\hline$T_{\max } / P$ & $0.87 \pm 0.22$ & $0.83 \pm 0.33$ & $0.87 \pm 0.09$ & $0.84 \pm 0.09$ & $0.99^{\mathrm{c}}$ \\
\hline
\end{tabular}

IDC invasive ductal carcinoma, ILC invasive lobular carcinoma, Mixed invasive carcinoma with presence of lobular and ductal components, $I M C$ invasive mucinous carcinoma, $K^{\text {trans }}$ contrast influx rate $(\mathrm{mL} / \mathrm{g} / \mathrm{min}), k_{e p}$ contrast efflux rate $\left(\mathrm{min}^{-1}\right), v_{e}$ fractional volume of extravascular-extracellular space, $v_{p}$ plasma fractional volume, $A D C$ apparent diffusion coefficient $\left(\mathrm{mm}^{2} / \mathrm{s}\right), K_{i}^{18} \mathrm{~F}$-FMISO influx rate $\left(\mathrm{mL} / \mathrm{cm}^{3} / \mathrm{min}\right), \% H F$ percentage hypoxic fraction, $S U V$ standardised uptake value $(\mathrm{g} / \mathrm{mL}), T_{\max } / M$ maximum tumour-to-muscle ratio, $T_{\max } / P$ maximum tumour-to-plasma ratio

${ }^{a}$ Mood's median test

${ }^{\mathrm{b}}$ Mann-Whitney $U$ test for malignancies of type IDC and ILC only (mixed and IMC lesions were not included in the comparison)

${ }^{\mathrm{c}}$ One-way analysis of variance (ANOVA) 


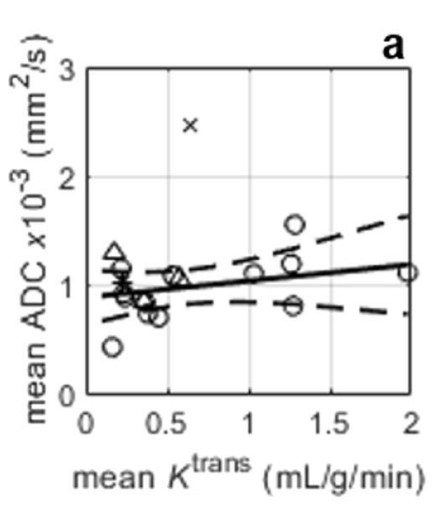

a

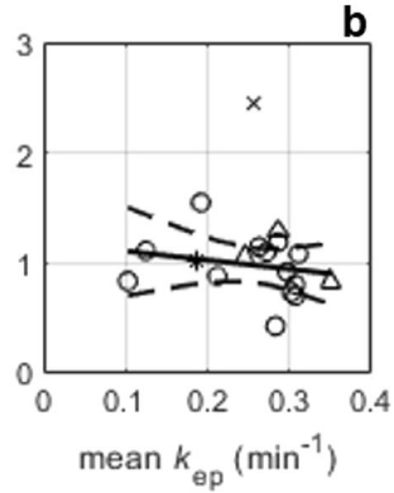

Fig. 4 Apparent diffusion coefficient (ADC) vs. DCE-MRI parameters: a contrast influx rate, $K^{\text {trans }} ; \mathbf{b}$ contrast efflux rate, $k_{\mathrm{ep}} ; \mathbf{c}$ fractional volume of extravascular-extracellular space, $v_{\mathrm{e}} ; \mathbf{d}$ plasma fractional volume, $v_{\mathrm{p}}$.

and perfusion has been previously documented, with the coexistence of hypoxic and hyperperfused tumour sub-volumes [39]. Various biological mechanisms, including hypoxiainduced angiogenesis, interstitial fluid pressure, a fluctuating haemodynamic response, increased oxygen diffusion distances from the microvasculature and the presence of longitudinal oxygen gradients across tumour vessels, have all been proposed to explain the occurrence of hypoxia in highly perfused areas [40, 41]. Thus, although the general trend of our results would support the widely accepted view that hypoxia develops in hypoperfused breast tumours, the diverse relationships observed in individual tumour sub-volumes indicate heterogeneity in hypoxia-perfusion patterns and reflect the variety of pathophysiological mechanisms occurring in cancers.

The weak relationship between PET hypoxia parameters with $k_{\text {ep }}$ suggests that the degree of tumour hypoxia is more strongly influenced by vascular flow rather than vessel
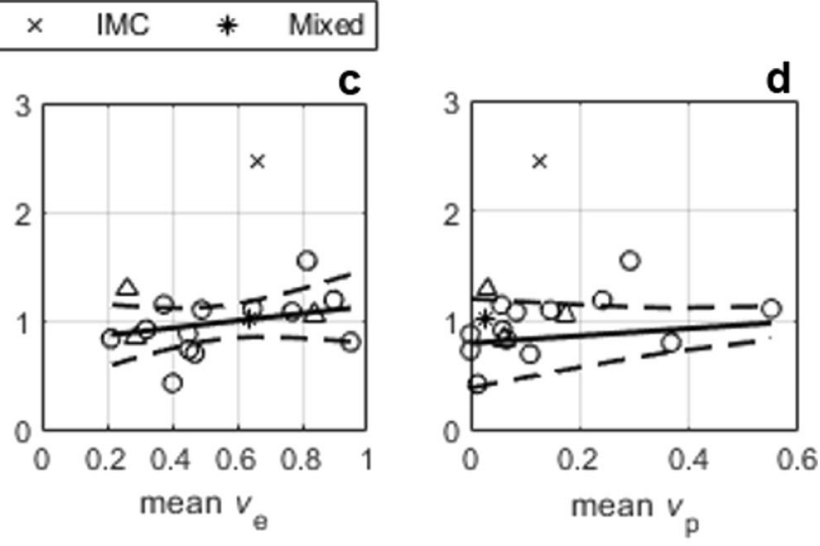

IDC, invasive ductal carcinoma; ILC, invasive lobular carcinoma; IMC, invasive mucinous carcinoma; Mixed, carcinoma of mixed ductal and lobular type

permeability. Li et al [42] have previously suggested that $k_{\mathrm{ep}}$ is a much more sensitive measure of vessel permeability than $K^{\text {trans }}$, as the latter represents a combined measure of blood flow, vessel permeability and capillary-surface area. Our findings broadly agree with previous research in cervical and head-and-neck carcinomas, which illustrated weaker correlations between hypoxia and permeability-surface-area product than between hypoxia and blood flow [37, 43]. The relationship between $K^{\text {trans }}$ and regional hypoxia observed in our study suggests this is due to fluctuations in tumour vascular flow rather than capillary permeability.

No or weak positive correlations were found between static ${ }^{18} \mathrm{~F}$-FMISO parameters $\left(\mathrm{SUV}_{\text {mean }}, \mathrm{SUV}_{\max }, T_{\max } / M, T_{\max } / P\right)$ and DCE-MRI metrics. In contrast, in human head-and-neck cancer, where hypoxia is often marked, ${ }^{18} \mathrm{~F}$-FMISO SUV measurements were negatively correlated with both $K^{\text {trans }}$ and $k_{\mathrm{ep}}$ [20]. A plausible explanation for this disparity is the
Fig. $5{ }^{18}$ F-FMISO-PET parameters vs. apparent diffusion coefficient (ADC): (a) influx rate $K_{\mathrm{i}}$ and (b) hypoxic fraction (\%). IDC, invasive ductal carcinoma; ILC, invasive lobular carcinoma; IMC, invasive mucinous carcinoma; Mixed, carcinoma of mixed ductal and lobular type

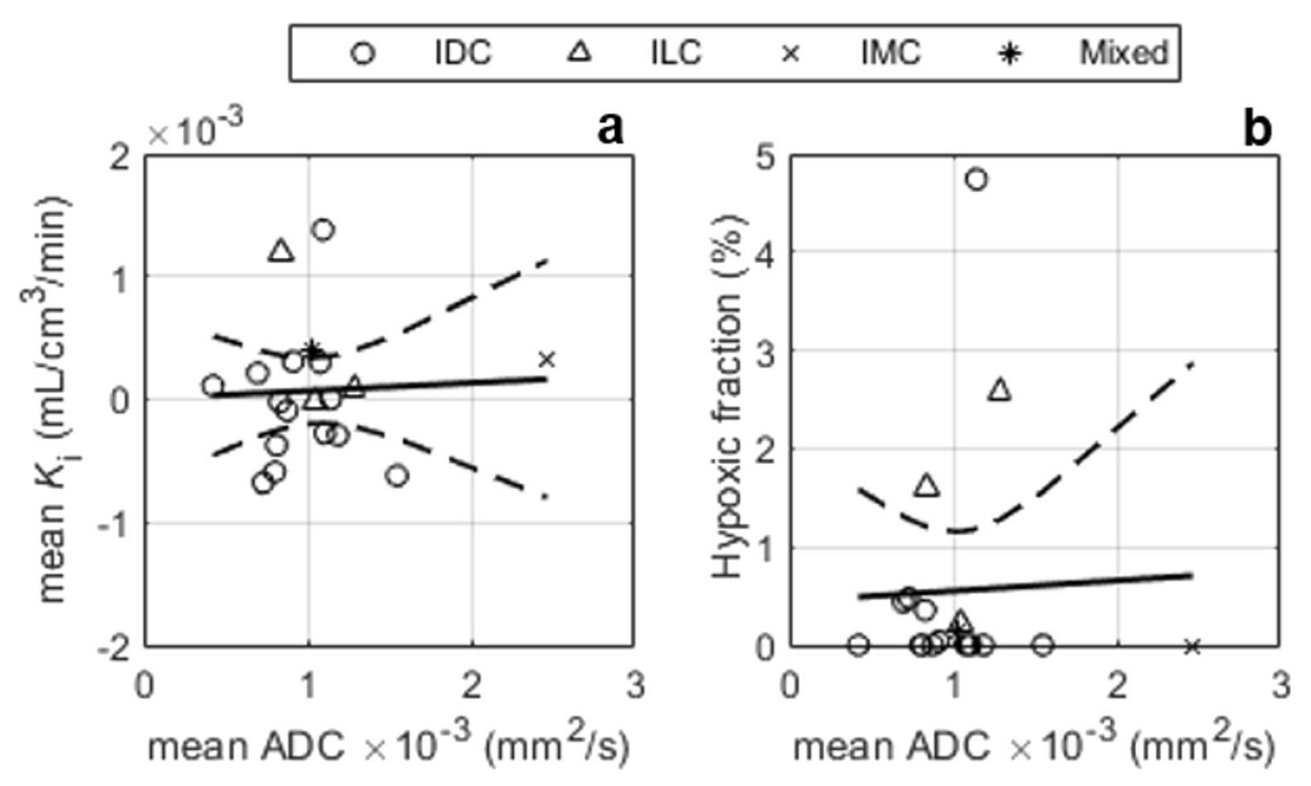




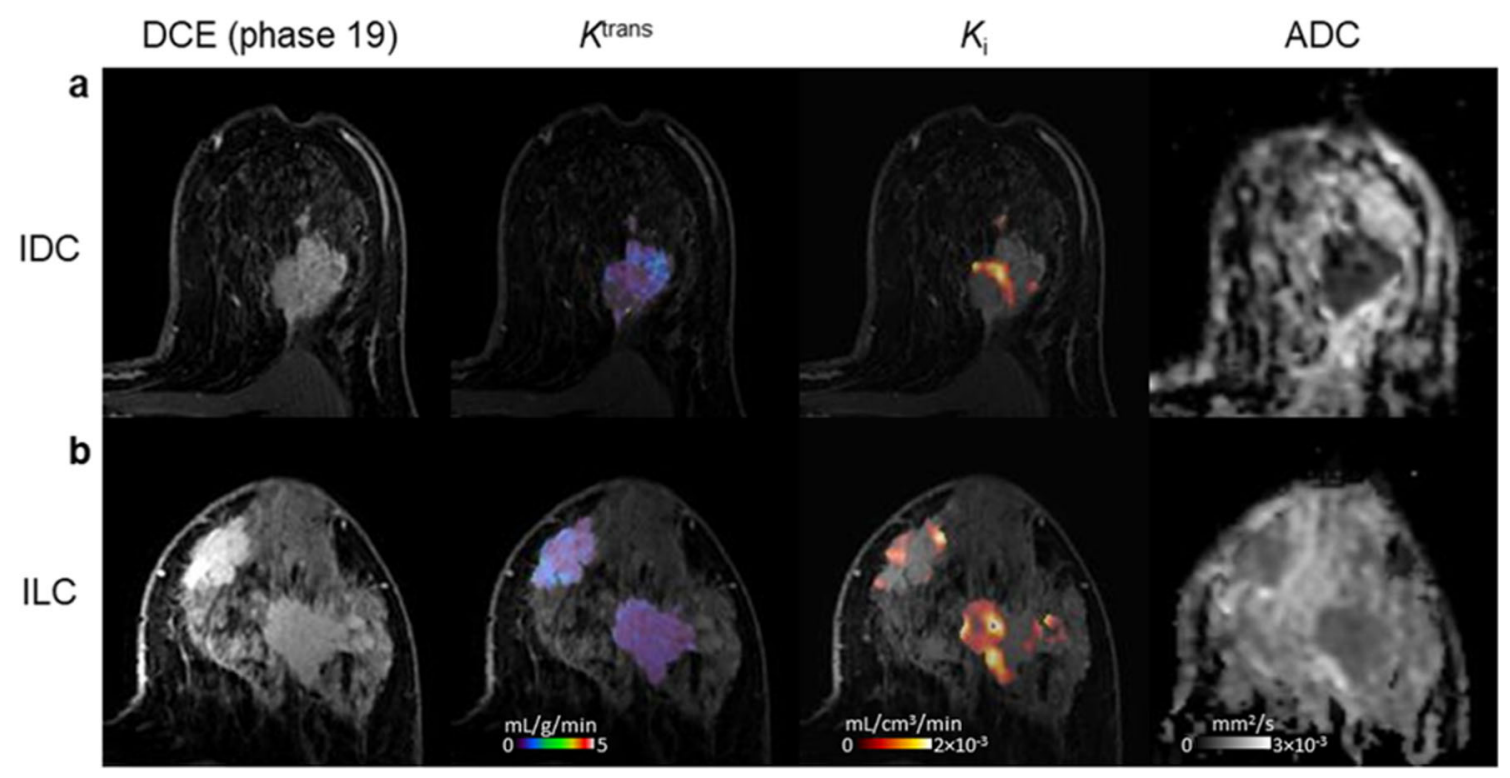

Fig. 6 Axial images of two patients with: a invasive ductal carcinoma (IDC); b invasive lobular carcinoma (ILC). (Left-to-right) DCE-MRI image at peak enhancement; $K^{\text {trans }}$ map representing tumour perfusion for the lesion ROI overlaid on the peak-enhancing DCE-MRI image; $K_{\mathrm{i}}$ map

higher level of hypoxia typically encountered in head-andneck cancer, which will lead to uptake values being more dominated by hypoxia-specific ${ }^{18} \mathrm{~F}$-FMISO trapping rather than non-specific tracer accumulation. Due to the higher contribution of non-specific ${ }^{18} \mathrm{~F}$-FMISO accumulation at low hypoxia levels [44], the use of uptake values in cancers without marked hypoxia may not accurately reveal relationships between hypoxia and perfusion.

No significant correlation was observed between PET hypoxia parameters and tumour grade or subtype. Our sample size of non-IDC cases was small for evaluating the impact of histology on tumour hypoxic status, but the presence of non-zero hypoxic fractions was observed in all histological subtypes studied. Hypoxic fractions and higher $K_{\mathrm{i}}$ were noted in both grade 2 and 3 tumours, and less so in grade 1 cancers. These findings are concordant with previously reported small differences in hypoxia between low- and high-grade breast malignancy [2].

Correlations between DCE-MRI functional parameters and pathological size or MR tumour diameter yielded moderate negative relationships and conversely positive associations between ${ }^{18}$ F-FMISO-PET hypoxia parameters and size. The size-related hypoxia changes could be ascribed to diffusionlimited hypoxia, concomitant perfusion decreases or increased interstitial fluid pressure [45].

$\mathrm{ADC}$ has been shown to inversely correlate with cellular density [46], and therefore, a reduction in ADC should theoretically be accompanied by an increase in tumour hypoxia. Our findings indicated no association between ADC and PET hypoxia parameters. This result could be explained by the molecular subtype of lesions in our sample, which representing tumour hypoxia for the lesion ROI overlaid on the DCEMRI image at peak enhancement; ADC map. $K^{\text {trans }}$, contrast influx rate $(\mathrm{mL} / \mathrm{g} / \mathrm{min}) ; K_{\mathrm{i}},{ }^{18} \mathrm{~F}$-FMISO influx rate $\left(\mathrm{mL} / \mathrm{cm}^{3} / \mathrm{min}\right) ; \mathrm{ADC}$, apparent diffusion coefficient $\left(\mathrm{mm}^{2} / \mathrm{s}\right)$

predominantly consisted of ER-positive/HER2-negative cancers. Due to lower blood flow, ER-positive or HER2-negative lesions exhibit lower ADC values than ER-negative or HER2positive cancers $[47,48]$. As ADC is affected not only by tissue cellularity but several pathophysiologic processes including blood flow, membrane permeability and the geometric architecture of the interstitial space $[49,50]$, it is likely that the lack of association between the PET hypoxia parameters and ADC is a consequence of the combined effect of cellularity, perfusion and microvessel structure on ADC. This assertion is further supported by the weak correlations between DCE-MRI indices and ADC observed in this study. It should be noted, however, that inconsistent correlations between ADC and DCE-MRI parameters have been reported in tumours, including breast cancer [51-53].

We calculated hypoxic fractions based on a specific parameter for hypoxia namely influx rate constant $K_{\mathrm{i}}$. Despite the higher variability associated with kinetic parameter estimates, our choice was based on two considerations. First, several authors have reported lack of correlation between ${ }^{18} \mathrm{~F}$ FMISO uptake ratios and $\mathrm{pO}_{2}$ measurements casting doubt on the accuracy of thresholds derived from static PET imaging for hypoxic quantification $[54,55]$. Kinetic parameters, including $K_{\mathrm{i}}$, have provided superior correlations with physiological measures of hypoxia from $\mathrm{pO}_{2}$ histography and immunohistochemistry $[54,55]$. Second, these thresholds have mostly been defined on measurements from head-and-neck cancers and are not necessarily applicable to other tumour types, including breast cancer.

The main limitations of our study are the small sample size and that the majority of cancers were HR-positive ductal 
carcinomas. Though our findings cannot be generalised to the full spectrum of histological/molecular subtypes encountered in breast cancer, our study indicates the presence of hypoxia in all histological subtypes studied independent of nuclear grade. While the majority of lesions $(56 \%)$ examined were found to be non-hypoxic, it should be noted that breast tumours are generally less hypoxic than cancers of the head and neck, cervix or lung and show greater variability in hypoxia among molecular subtypes, with basal-like subtypes being the most hypoxic [56].

Our demonstration of in vivo simultaneous measurement of perfusion and hypoxia is clinically important for three reasons. First, previous reports have indicated that tumours with a high hypoxia-perfusion ratio (i.e. hypoxia due to low perfusion) have a poorer prognosis and suboptimal treatment response $[57,58]$. In breast cancer, studies have described differences in the response to perfusion-related hypoxic exposure between molecular subtypes [59,60], emphasising the need for combined hypoxia-perfusion measurements to provide more accurate prognostic information or tailor treatment. Second, preoperative radiotherapy or radiochemotherapy regimes in early or locally advanced breast cancer have reported beneficial clinical outcomes [61, 62]. Hypoxia and hypoperfusion are known to reduce the effectiveness of radiotherapy and chemotherapy, and the hypoxia-perfusion status of tumours at baseline could allow optimisation of these regimens. Third, tumour hypoxia can occur independently of hypoperfusion as evidenced in the oncology literature [39, 40, 57, 58] and our findings. As such, the data presented here can be viewed as providing further indication of the benefit of noninvasive multimodal assessment of the tumour microenvironment for disease characterisation.

In conclusion, we found a negative relationship between tumour hypoxia, measured by ${ }^{18} \mathrm{~F}$-FMISO-PET, and markers of perfusion and vascular function from DCE-MRI, endorsing the hypothesis of perfusion-driven hypoxia in breast cancer. No associations were observed between ${ }^{18} \mathrm{~F}$-FMISO-PET parameters and tumour histology or grade, but hypoxic fractions increased with lesion size. The intratumoural heterogeneity observed in hypoxia and perfusion images is consistent with the known complex relationship between perfusion and the hypoxic tumour micromilieu. The combined hypoxiaperfusion status of tumours may need to be considered in determining treatment efficacy or informing therapy selection in breast cancer, which could be achieved using simultaneous multimodality imaging as reported here.

Acknowledgements The authors would like to thank the Radiopharmaceutical Unit, Wolfson Brain Imaging Centre, University of Cambridge, for the provision of ${ }^{18} \mathrm{~F}$-FMISO; the radiographic team and research nurses at the Wolfson Brain Imaging Centre and the Department of Radiology, University of Cambridge, as well as the clinical staff at the Cambridge Breast Unit, Cambridge University Hospitals NHS Foundation Trust.
Funding information This study was co-funded by Cancer Research UK (CRUK) - Cambridge Institute (CCCIT02) and the National Institute for Health Research (NIHR) Cambridge Biomedical Research Centre (BRC). J. C. Carmona-Bozo is supported by the Vargas Scholarship, Darwin College, University of Cambridge. R. Manavaki, M. J. Graves and A. J. Patterson are supported by NIHR Cambridge BRC. F. J. Gilbert is a Senior Investigator at NIHR Cambridge BRC. The University of Cambridge PET/MR facility was funded by the Medical Research Council (MRC), UK. The views expressed are those of the authors and not necessarily those of the NIHR or the Department of Health and Social Care, UK.

\section{Compliance with ethical standards}

Guarantor The scientific guarantor of this publication is Professor Fiona J Gilbert.

Conflict of interest The authors declare relationships with the following companies: FJG has research collaborations with Bayer Healthcare, GE Healthcare, Hologic Inc., and a consultancy arrangement with Google.

Statistics and biometry No complex statistical methods were necessary for this paper.

Informed consent Written informed consent was obtained from all subjects (patients) in this study.

Ethical approval Institutional Review Board approval was obtained. All procedures performed involving human participants were in accordance with the ethical standards of a National Research Ethics Committee (NRES Committee East of England - Cambridge Central; 14/EE/0145) and the Administration of Radioactive Substances Advisory Committee (ARSAC), UK.

\section{Methodology \\ - prospective \\ - observational \\ - performed at one institution}

Open Access This article is licensed under a Creative Commons Attribution 4.0 International License, which permits use, sharing, adaptation, distribution and reproduction in any medium or format, as long as you give appropriate credit to the original author(s) and the source, provide a link to the Creative Commons licence, and indicate if changes were made. The images or other third party material in this article are included in the article's Creative Commons licence, unless indicated otherwise in a credit line to the material. If material is not included in the article's Creative Commons licence and your intended use is not permitted by statutory regulation or exceeds the permitted use, you will need to obtain permission directly from the copyright holder. To view a copy of this licence, visit http://creativecommons.org/licenses/by/4.0/.

\section{References}

1. Vaupel P, Kallinowski F, Okunieff P (1989) Blood flow, oxygen and nutrient supply, and metabolic microenvironment of human tumors: a review. Cancer Res 49:6449-6465

2. Vaupel P, Schlenger K, Knoop C, Höckel M (1991) Oxygenation of human tumors: evaluation of tissue oxygen distribution in breast cancers by computerized $\mathrm{O}_{2}$ tension measurements. Cancer Res 51: $3316-3322$ 
3. Hohenberger P, Felgner C, Haensch W, Schlag PM (1998) Tumor oxygenation correlates with molecular growth determinants in breast cancer. Breast Cancer Res Treat 48:97-106

4. Helczynska K, Kronblad A, Jögi A et al (2003) Hypoxia promotes a dedifferentiated phenotype in ductal breast carcinoma in situ. Cancer Res 63:1441-1444

5. Cooper C, Liu GY, Niu YL, Santos S, Murphy LC, Watson PH (2004) Intermittent hypoxia induces proteasome-dependent downregulation of estrogen receptor $\alpha$ in human breast carcinoma. Clin Cancer Res 10:8720-8727

6. Semenza GL (2016) The hypoxic tumor microenvironment: a driving force for breast cancer progression. Biochim Biophys Acta 1863:382-391

7. Chia SK, Wykoff CC, Watson PH et al (2001) Prognostic significance of a novel hypoxia-regulated marker, carbonic anhydrase IX, in invasive breast carcinoma. J Clin Oncol 19:3660-3668

8. Generali D, Berruti A, Brizzi MP et al (2006) Hypoxia-inducible factor- $1 \alpha$ expression predicts a poor response to primary chemoendocrine therapy and disease-free survival in primary human breast cancer. Clin Cancer Res 12:4562-4568

9. Dewhirst MW, Cao Y, Moeller B (2008) Cycling hypoxia and free radicals regulate angiogenesis and radiotherapy response. Nat Rev Cancer 8:425-437

10. Kimura H, Braun RD, Ong ET et al (1996) Fluctuations in red cell flux in tumor microvessels can lead to transient hypoxia and reoxygenation in tumor parenchyma. Cancer Res 56:5522-5228

11. Bristow RG, Hill RP (2008) Hypoxia and metabolism. Hypoxia, DNA repair and genetic instability. Nat Rev Cancer 8:180-192

12. Turnbull LW (2009) Dynamic contrast-enhanced MRI in the diagnosis and management of breast cancer. NMR Biomed 22:28-39

13. Partridge SC, Nissan N, Rahbar H, Kitsch AE, Sigmund EE (2017) Diffusion-weighted breast MRI: clinical applications and emerging techniques. J Magn Reson Imaging 45:337-355

14. Krohn KA, Link JM, Mason RP (2008) Molecular imaging of hypoxia. J Nucl Med 49:129S-148S

15. Quintela-Fandino M, Lluch A, Manso L et al $(2017){ }^{18} \mathrm{~F}$ fluoromisonidazole PET and activity of neoadjuvant nintedanib in early HER2-negative breast cancer: a window-of-opportunity randomized trial. Clin Cancer Res 23:1432-1441

16. Ueda S, Saeki T, Osaki A, Yamane T, Kuji I (2017) Bevacizumab induces acute hypoxia and cancer progression in patients with refractory breast cancer: multimodal functional imaging and multiplex cytokine analysis. Clin Cancer Res 23:5769-5778

17. Cheng J, Lei L, Xu J et al (2013) ${ }^{18}$ F-fluoromisonidazole PET/CT: a potential tool for predicting primary endocrine therapy resistance in breast cancer. J Nucl Med 54:333-340

18. Asano A, Ueda S, Kuji I et al (2018) Intracellular hypoxia measured by ${ }^{18} \mathrm{~F}$-fluoromisonidazole positron emission tomography has prognostic impact in patients with estrogen receptor-positive breast cancer. Breast Cancer Res 20:78

19. Andrzejewski P, Wengert G, Helbich TH et al (2019) Sequential $\left[{ }^{18} \mathrm{~F}\right]$ FDG $-\left[{ }^{18} \mathrm{~F}\right]$ FMISO PET and multiparametric MRI at $3 \mathrm{~T}$ for insights into breast cancer heterogeneity and correlation with patient outcomes: first clinical experience. Contrast Media Mol Imaging. https://doi.org/10.1155/2019/1307247

20. Jansen JF, Schöder H, Lee NY et al (2010) Noninvasive assessment of tumor microenvironment using dynamic contrast-enhanced magnetic resonance imaging and ${ }^{18} \mathrm{~F}$-fluoromisonidazole positron emission tomography imaging in neck nodal metastases. Int J Radiat Oncol Biol Phys 77:1403-1410

21. Gerstner ER, Zhang Z, Fink JR et al (2016) ACRIN 6684: assessment of tumor hypoxia in newly diagnosed glioblastoma using ${ }^{18} \mathrm{~F}$ FMISO PET and MRI. Clin Cancer Res 22:5079-5086

22. Pinker K, Andrzejewski P, Baltzer P et al (2016) Multiparametric $\left[{ }^{18} \mathrm{~F}\right]$ Fluorodeoxyglucose $/\left[{ }^{18} \mathrm{~F}\right]$ Fluoromisonidazole positron emission tomography/magnetic resonance imaging of locally advanced cervical cancer for the non-invasive detection of tumor heterogeneity: a pilot study. PLoS One 11:e0155333. https://doi.org/10.1371/ journal.pone. 0155333

23. Simoncic U, Leibfarth S, Welz S et al (2017) Comparison of DCEMRI kinetic parameters and FMISO-PET uptake parameters in head and neck cancer patients. Med Phys 44:23582368

24. Taylor E, Zhou J, Lindsay P et al (2020) Quantifying reoxygenation in pancreatic cancer during stereotactic body radiotherapy. Sci Rep 10:1638

25. Rasey JS, Grunbaum Z, Magee S et al (1987) Characterization of radiolabeled fluoromisonidazole as a probe for hypoxic cells. Radiat Res 111:292-304

26. Muzi M, Peterson LM, O'Sullivan JN et al (2015) ${ }^{18} \mathrm{~F}$ fluoromisonidazole quantification of hypoxia in human cancer patients using image-derived blood surrogate tissue reference regions. J Nucl Med 56:1223-1228

27. Patlak CS, Blasberg RG, Fenstermacher JD (1983) Graphical evaluation of blood-to-brain transfer constants from multiple-time uptake data. J Cereb Blood Flow Metab 3:1-7

28. Belton M, Brilha S, Manavaki R et al (2016) Hypoxia and tissue destruction in pulmonary TB. Thorax 71:1145-1153

29. Koh WJ, Rasey JS, Evans ML et al (1992) Imaging of hypoxia in human tumors with [F-18]fluoromisonidazole. Int J Radiat Oncol Biol Phys 22:199-212

30. Rasey JS, Koh WJ, Evans ML et al (1996) Quantifying regional hypoxia in human tumors with positron emission tomography of $\left[{ }^{18} \mathrm{~F}\right]$ fluoromisonidazole: a pretherapy study of 37 patients. Int $\mathrm{J}$ Radiat Oncol Biol Phys 36:417-428

31. Bruehlmeier M, Roelcke U, Schubiger PA, Ametamey SM (2004) Assessment of hypoxia and perfusion in human brain tumors using PET with ${ }^{18} \mathrm{~F}$-fluoromisonidazole and ${ }^{15} \mathrm{O}-\mathrm{H}_{2} \mathrm{O}$. J Nucl Med 45: $1851-1859$

32. Bedair R, Graves MJ, Patterson AJ et al (2016) Effect of radiofrequency transmit field correction on quantitative dynamic contrastenhanced MR imaging of the breast at 3.0T. Radiology 279:368377

33. Banelli B, Casciano I, Di Vinci A et al (2010) Pathological and molecular characteristics distinguishing contralateral metastatic from new primary breast cancer. Ann Oncol 21:1237-1242

34. Tofts PS, Brix G, Buckley DL et al (1999) Estimating kinetic parameters from dynamic contrast-enhanced $\mathrm{T}_{1}$-weighted MRI of a diffusable tracer: standardized quantities and symbols. J Magn Reson Imaging 10:223-232

35. Baltzer P, Mann RM, lima M et al (2020) Diffusion-weighted imaging of the breast-a consensus and mission statement from the EUSOBI International Breast Diffusion-Weighted Imaging working group. Eur Radiol 30:1436-1450

36. Liney GP, Gibbs P, Hayes C, Leach MO, Turnbull LW (1999) Dynamic contrast-enhanced MRI in the differentiation of breast tumors: user-defined versus semi-automated region-of-interest analysis. J Magn Reson Imaging 10:945-949

37. Donaldson SB, Betts G, Bonington SC et al (2011) Perfusion estimated with rapid dynamic contrast-enhanced magnetic resonance imaging correlates inversely with vascular endothelial growth factor expression and pimonidazole staining in head-and-neck cancer: a pilot study. Int J Radiat Oncol Biol Phys 81:1176-1183

38. Cooper R, Carrington BM, Loncaster J et al (2000) Tumour oxygenation levels correlate with dynamic contrast-enhanced magnetic resonance imaging parameters in carcinoma of the cervix. Radiother Oncol 57:53-59

39. Grkovski M, Schöder H, Lee NY et al (2017) Multiparametric imaging of tumor hypoxia and perfusion with ${ }^{18} \mathrm{~F}$ fluoromisonidazole dynamic PET in head and neck cancer. J Nucl Med 58:1072-1080 
40. Dewhirst MW, Navia IC, Brizel DM, Willett C, Secomb TW (2007) Multiple etiologies of tumor hypoxia require multifaceted solutions. Clin Cancer Res 13:375-377

41. Beaney RP, Lammertsma AA, Jones T, McKenzie CG, Halnan KE (1984) Positron emission tomography for in-vivo measurement of regional blood flow, oxygen utilisation, and blood volume in patients with breast carcinoma. Lancet 1(8369):131-134

42. Li SP, Padhani AR, Taylor NJ et al (2011) Vascular characterisation of triple negative breast carcinomas using dynamic MRI. Eur Radiol 21:1364-1373

43. Dickie BR, Rose CJ, Kershaw LE et al (2017) The prognostic value of dynamic contrast-enhanced MRI contrast agent transfer constant $K^{\text {trans }}$ in cervical cancer is explained by plasma flow rather than vessel permeability. Br J Cancer 116:1436-1443

44. Nunn A, Linder K, Strauss HW (1995) Nitroimidazoles and imaging hypoxia. Eur J Nucl Med 22:265-280

45. Dadiani M, Margalit R, Sela N, Degani H (2004) High-resolution magnetic resonance imaging of disparities in the transcapillary transfer rates in orthotopically inoculated invasive breast tumors. Cancer Res 64:3155-3161

46. Surov A, Meyer HJ, Wienke A (2017) Correlation between apparent diffusion coefficient (ADC) and cellularity is different in several tumors: a meta-analysis. Oncotarget 8:59492-59499

47. Jeh SK, Kim SH, Kim HS et al (2011) Correlation of the apparent diffusion coefficient value and dynamic magnetic resonance imaging findings with prognostic factors in invasive ductal carcinoma. $\mathrm{J}$ Magn Reason Imaging 3:102-109

48. Martincich L, Deantoni V, Bertotto I et al (2012) Correlations between diffusion-weighted imaging and breast cancer biomarkers. Eur Radiol 22:1519-1528

49. Le Bihan D (1995) Molecular diffusion, tissue microdynamics and microstructure. NMR Biomed 8:375-386

50. Le Bihan D (2013) Apparent diffusion coefficient and beyond: what diffusion MR imaging can tell us about tissue structure. Radiology 268:318-322

51. Chu JP, Mak HK, Yau KK et al (2012) Pilot study on evaluation of any correlation between MR perfusion $\left(\mathrm{K}^{\text {trans }}\right)$ and diffusion (apparent diffusion coefficient) parameters in brain tumors at 3 Tesla. Cancer Imaging 12:1-6

52. Arlinghaus LR, Li X, Rahman AR et al (2011) On the relationship between the apparent diffusion coefficient and extravascular extracellular volume fraction in human breast cancer. Magn Reson Imaging 29:630-638

53. Yankeelov TE, Lepage M, Chakravarthy A et al (2007) Integration of quantitative DCE-MRI and ADC mapping to monitor treatment response in human breast cancer: initial results. Magn Reson Imaging 25:1-13

54. Kelada OJ, Rockwell S, Zheng MQ et al (2017) Quantification of tumor hypoxic fractions using positron emission tomography with $\left[{ }^{18} \mathrm{~F}\right]$ fluoromisonidazole $\left(\left[{ }^{18} \mathrm{~F}\right] \mathrm{FMISO}\right)$ kinetic analysis and invasive oxygen measurements. Mol Imaging Biol 19:893-902

55. Shi K, Bayer C, Astner ST et al (2017) Quantitative analysis of $\left[{ }^{18}\right.$ F]FMISO PET for tumor hypoxia: correlation of modeling results with immunohistochemistry. Mol Imaging Biol 19:120-129

56. Bhandari V, Hoey C, Liu LY et al (2019) Molecular landmarks of tumor hypoxia across cancer types. Nat Genet 51:308-318

57. Lehtiö K, Eskola O, Viljanen T et al (2004) Imaging perfusion and hypoxia with PET to predict radiotherapy response in head-andneck cancer. Int J Radiat Oncol Biol Phys 59:971-982

58. Thorwarth D, Eschmann SM, Scheiderbauer J, Paulsen F, Alber M (2005) Kinetic analysis of dynamic ${ }^{18} \mathrm{~F}$-fluoromisonidazole PET correlates with radiation treatment outcome in head-and-neck cancer. BMC Cancer 5:152

59. Harrison H, Rogerson L, Gregson HJ, Brennan KR, Clarke RB, Landberg G (2013) Contrasting hypoxic effects on breast cancer stem cell hierarchy is dependent on ER- $\alpha$ status. Cancer Res 73: $1420-1433$

60. von Minckwitz G, Eidtmann H, Rezai M et al (2012) Neoadjuvant chemotherapy and bevacizumab for HER2-negative breast cancer. N Engl J Med 366:299-309

61. Poleszczuk J, Luddy K, Chen L et al (2017) Neoadjuvant radiotherapy of early-stage breast cancer and long-term disease-free survival. Breast Cancer Res 19:75

62. Brackstone M, Palma D, Tuck AB et al (2017) Concurrent neoadjuvant chemotherapy and radiation therapy in locally advanced breast cancer. Int J Radiat Oncol Biol Phys 99:769-776

Publisher's note Springer Nature remains neutral with regard to jurisdictional claims in published maps and institutional affiliations. 